\title{
OPEN SOURCE APPROACH TO URBAN GROWTH SIMULATION
}

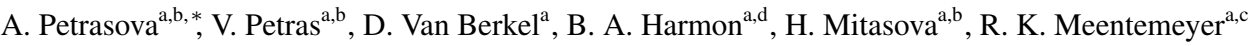 \\ ${ }^{a}$ Center for Geospatial Analytics, North Carolina State University, USA - dbvanber@ncsu.edu \\ ${ }^{\mathrm{b}}$ Department of Marine, Earth, and Atmospheric Sciences, North Carolina State University, USA - (vpetras, akratoc, hmitaso)@ncsu.edu \\ ${ }^{\mathrm{c}}$ Department of Forestry and Environmental Resources, North Carolina State University, USA - rkmeente@ncsu.edu \\ d Department of Landscape Architecture, North Carolina State University, USA - baharmon@ ncsu.edu
}

Commission VII, SpS10 - FOSS4G: FOSS4G Session (coorganized with OSGeo)

KEY WORDS: GRASS GIS, FUTURES, urbanization, land change, open science, simulation

\begin{abstract}
:
Spatial patterns of land use change due to urbanization and its impact on the landscape are the subject of ongoing research. Urban growth scenario simulation is a powerful tool for exploring these impacts and empowering planners to make informed decisions. We present FUTURES (FUTure Urban - Regional Environment Simulation) - a patch-based, stochastic, multi-level land change modeling framework as a case showing how what was once a closed and inaccessible model benefited from integration with open source GIS. We will describe our motivation for releasing this project as open source and the advantages of integrating it with GRASS GIS, a free, libre and open source GIS and research platform for the geospatial domain. GRASS GIS provides efficient libraries for FUTURES model development as well as standard GIS tools and graphical user interface for model users. Releasing FUTURES as a GRASS GIS add-on simplifies the distribution of FUTURES across all main operating systems and ensures the maintainability of our project in the future. We will describe FUTURES integration into GRASS GIS and demonstrate its usage on a case study in Asheville, North Carolina. The developed dataset and tutorial for this case study enable researchers to experiment with the model, explore its potential or even modify the model for their applications.
\end{abstract}

\section{INTRODUCTION}

Population growth in cities worldwide drives changes in land use often negatively impacting the environments in which people live and undermining the resilience of local ecosystems. The need to understand the trade-offs urban planners are facing gave rise to a number of different land change simulation models, which proved to be powerful tools for exploring alternative scenarios and their impacts on various aspects of human-environmental systems (Chaudhuri and Clarke, 2013, Verburg et al., 2002, Sohl et al., 2007, Waddell, 2002). Despite the influence of the spatial structure and connectivity of urbanizing landscapes on biodiversity, water quality, or flood risks (Alberti, 2005), most urban growth models are based on cell-level conversions and have not focused on generating realistic spatial structures across scales (Jantz and Goetz, 2005). To bridge the gap between cell- and object-based representation, we developed FUTURES (FUTure Urban-Regional Environment Simulation), a patch-based, multilevel modeling framework for simulating the emergence of landscape spatial structure in urbanizing regions (Meentemeyer et al., 2013). The FUTURES model was successfully applied in several cases including a study of land development dynamics in the rapidly expanding metropolitan region of Charlotte, North Carolina (Meentemeyer et al., 2013) and an analysis of the impacts of urbanization on natural resources under different conservation strategies (Dorning et al., 2015). Most recently, FUTURES was coupled with ecosystem services models to examine the impacts of projected urbanization and urban pattern on several ecosystem services and their trade-offs (Shoemaker, 2016, Pickard et al., in prep.).

In order to study the complex interactions between human and natural systems, interdisciplinary researchers are coupling existing simulation models. Land change modeling plays often a cru-

\footnotetext{
${ }^{*}$ Corresponding author
}

cial role in these coupled models. Previous case studies with FUTURES have demonstrated that the model can be applied to a wide range of cases with different study systems and aims. The initial implementation of model, however, was a prototype that was not ready to be shared with scientific community. The model accumulated too much "technical depth" (Easterbrook, 2014) during its initial development, making it difficult to add new features or run the simulation at larger scales. In order to continue adding new capabilities to FUTURES and to promote its usage both inside and outside of the land use community, we decided to revise the implementation of the FUTURES model and develop a new version which would be (a) more efficient and scalable, (b) as easy to use as possible for a wider audience and (c) fully open source and maintainable in the long run. To achieve these goals we decided that instead of keeping FUTURES as a standalone application, we would take advantage of existing geospatial software and integrate FUTURES into open source GRASS GIS (Neteler and Mitasova, 2008). By using GRASS GIS' efficient geospatial libraries we can develop better and higher-level code. Providing open source software to the scientific community entails more than just releasing the actual code - documentation, tutorials, installation instructions, binaries and support are also needed and require considerable effort. Without this effort, models cannot be practically used by other researchers. By using GRASS GIS' existing infrastructure we could focus on developing the actual materials instead of managing our own server infrastructure.

In this article we present a new version of the FUTURES urban growth model that is available as the r.futures module set from the GRASS GIS add-on repository. This new version of FUTURES streamlines data processing, provides opportunities to study urbanization on mega-regional scales, and allows for more reproducible research in the land change community. We demonstrate this new version of FUTURES with a case study of the Asheville metropolitan area in North Carolina, USA. 


\section{FUTURES MODEL}

FUTure Urban-Regional Environment Simulation is a stochastic, patched-based model for projecting landscape patterns of urban growth (Meentemeyer et al., 2013). FUTURES has a modular structure consisting of 3 main submodels: DEMAND, POTENTIAL and PGA (patch-growing algorithm), see Figure 1. Land conversion is driven by projected population demand computed by the DEMAND submodel, and is spatially defined by a probability surface derived by the POTENTIAL submodel from multiple environmental and socio-economic predictors. The population demand and the effects of land change drivers can vary in space by subregions, such as jurisdictional units, allowing projections across heterogeneous landscape. FUTURES main strength lies in realistically modeling the spatial structure of urban change by growing patches that are parameterized by size and compactness and calibrated using historical data. For a detailed explanation of FUTURES' components, please refer to Meentemeyer et al. (2013).

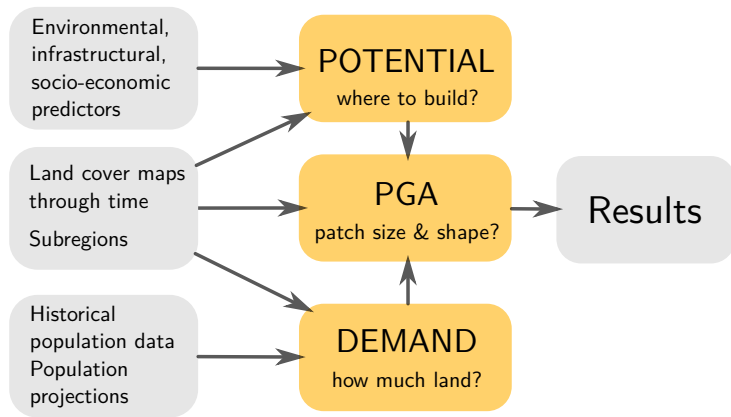

Figure 1: Simplified schema of FUTURES conceptual model with inputs and outputs in gray and submodels in yellow

The original implementation of FUTURES consisted mainly of the patch-growing algorithm, a standalone program written in a mixture of $\mathrm{C}$ and $\mathrm{C}++$. The PGA program itself utilized inefficient algorithms and required raster data in ASCII format as input leading to very slow initialization. The DEMAND submodel was computed in a spreadsheet and POTENTIAL coefficents were derived using $\mathrm{R}$ statistical software. No official implementation of these submodels existed so each researcher developed a different workflow. This made it difficult for peers to verify each other's work. Several scripts for calibrating patch characteristics derived with FRAGSTATS (McGarigal et al., 2012) existed, however these tools were written in an unnecessarily low-level language for a specific case using the author's directory layout.

When revising the original implementation of FUTURES we identified several issues which needed to be addressed. First, it is important to follow best practices for scientific computing (Wilson et al., 2014) including use of a versioning system, writing documentation and testing. We also wanted to minimize tasks that had been done manually in order to make the process more efficient and avoid errors that are often difficult to detect. When automating tasks we had to compromise between the flexibility and simplicity of the workflow. We also focused on making FUTURES scalable so that it can run large scale applications at a relatively fine spatial resolution. Finally, we designed FUTURES to be more user-friendly and easy to test so that anyone can confidently apply it to their research.

\section{INTEGRATION IN GRASS GIS}

GRASS GIS has had a long history as a platform for scientific models (Chemin et al., 2015). As an open source GIS used by researchers worldwide and one of the founding projects of OSGeo, GRASS GIS provides a stable environment for the development of scientific models for studying problems from various domains including geomorphology, hydrology, planetary science, landscape ecology, hazard mapping, archaeology, renewable energy and transportation. Thanks to the numerous scientist and developers who have been involved, GRASS GIS today provides a large spectrum of geospatial modules ranging from basic GIS functionality to highly specialized models. Most of the specialized tools are not part of standard GRASS GIS installations, but are easily accessible from the add-on repository.

There were multiple reasons for our decision to integrate FUTURES into GRASS GIS as an add-on. Some of these reasons were specific to FUTURES, but others apply to any spatial, scientific model. Integrating a model into GIS gives both users and developers a wide array of standard geospatial tools that simplify the implementation of a model, and streamline pre- and postprocessing and visualization. GRASS GIS provides model developers a raster library for highly efficient data reading and writing. This means that FUTURES no longer has to read in ASCII files, significantly reducing time needed for initialization. Furthermore, raster data from FUTURES simulations are efficiently compressed. Despite ever increasing disk space, it is still quite important to reduce the file size, especially for stochastic spatiotemporal simulations, which typically generate huge datasets. In order to achieve the best speed performance, most GRASS GIS functionality is implemented in C. Because of this, we could easily integrate FUTURES code, written in a mix of $\mathrm{C}$ and $\mathrm{C}++$, without major rewriting. For portability reasons we later decided to use the $\mathrm{C} 99$ standard. While $\mathrm{C}$ and $\mathrm{C}++$ are the preferred languages for computationally expensive algorithms, GRASS GIS also supports Python as the primary scripting language. This is crucial because FUTURES had many steps of data preparation that we were easily able to automate using Python scripting. Model developers can appreciate GRASS GIS' automatic generation of command line interfaces, Python interfaces and graphical user interfaces (GUI). Simply by defining options in C or Python modules we can call the same module from a GUI dia$\log$, a Python script or a Bash script. A graphical interface makes FUTURES easy to use, especially for users on the Windows platform. A Python or Bash interface, however, is needed for more advanced applications such as running FUTURES in parallel on a high performance computer. GRASS GIS provides infrastructure for publishing and distributing models to users on all major platforms. Models and tools in GRASS GIS's Add-on repository ${ }^{1}$ can be easily browsed and installed with their documentation, relieving researchers of the burden of maintaining such infrastructure.

\subsection{Implementation}

We implemented FUTURES as a set of GRASS GIS modules starting with a common prefix r.futures:

- r.futures.demand extrapolates the area of developed land from population trends and projections.

- r.futures.devpressure computes the development pressure predictor.

- r.futures.potential models the development probability surface through multi-level logistic regression.

- r.futures.calib calibrates patch sizes and shapes.

- r.futures.pga simulates urban development using the patch growing algorithm.

\footnotetext{
${ }^{1}$ https://grass.osgeo.org/download/addons/
} 
In addition, we implemented the add-on r.sample.category needed for the workflow. Since its functionality is not specific to FUTURES, we kept it separate. All of these add-ons can be conveniently installed from GRASS GIS using the GUI or command line $^{2}$. Each individual add-on has a manual page accessible both online and offline. Figure 2 shows FUTURES workflow and the inputs needed for each tool. In the following sections we describe the developed tools, their functionality and implementation in GRASS GIS.

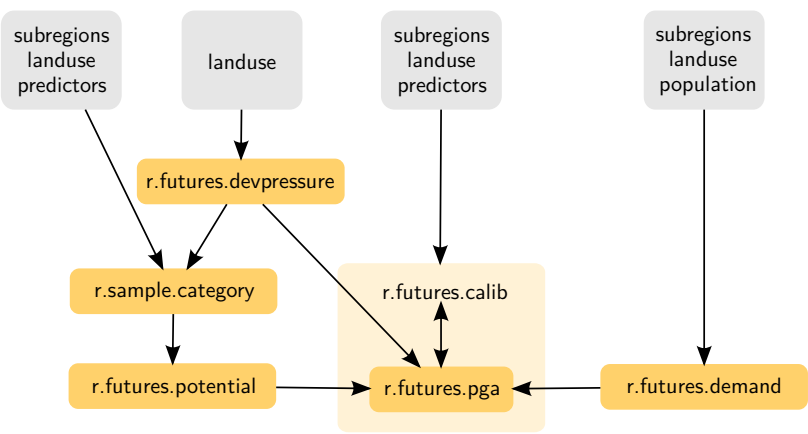

Figure 2: Diagram of FUTURES workflow showing how are r.futures modules (yellow boxes) chained and what are their input data (grey boxes). As indicated by the light yellow box, module r.futures.calib calls r.futures.pga.

3.1.1 r.futures.demand Based on historical land development and population growth, the DEMAND submodel (implemented as r.futures.demand) projects the rate of per capita land consumption for each year of the simulation and each subregion. This Python module uses GRASS GIS Python Scripting Library and the NumPy, SciPy and matplotlib libraries for scientific computing to approximate the relation between population and land consumption with a statistical model described by a linear, logarithmic or exponential curve. For example, a logarithmic relation means that a growing population requires less developed land per person over time. With enough data points, the module can select the best curve for each subregion based on residuals. The primary outputs are plain text files with tab-separated values representing the number of cells to be converted to developed land each year for each subregion. The module plots the resulting curves and projected points for each subregion (Figure 3) so that the results can be visually inspected. The module r.futures.demand provides a fast way to estimate the land demand given a large number of subregions with diverse population trends and thus allows us to quickly explore different population scenarios.

3.1.2 r.futures.devpressure Development pressure is one of the most important predictors of where development is likely to happen. For each cell it is computed as a distance decay function of neighboring developed cells (Meentemeyer et al., 2013). Compared to the tool previously used for computing development pressure, the new Python module r.futures.devpressure provides a faster and more efficient implementation by taking advantage of the existing GRASS GIS module r.mfilter written in C for moving window analysis with custom designed matrix filters. By precomputing the matrix of distances we avoid repeated distance computations resulting in faster processing. Because the new implementation is less memory intensive it can be used for larger regions than the previous tool.

3.1.3 r.futures.potential uses multilevel logistic regression to model development suitability based on environmental, infrastructural, and socio-economic predictors such as distance to roads or topographic slope. We randomly sample these predictors and

${ }^{2}$ g.extension r.futures

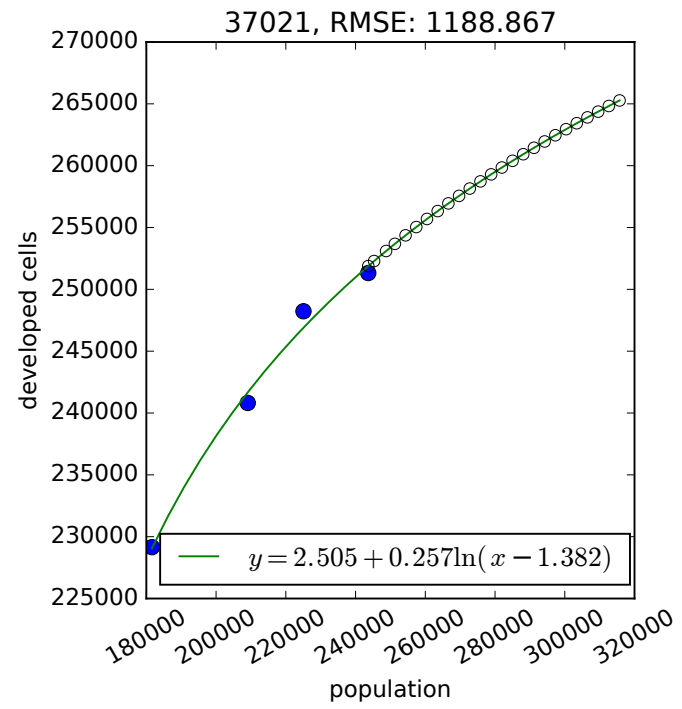

Figure 3: An example of r.futures.demand output plot showing the logarithmic relation between population and land consumption for the county with ID 37021. Observed data are showed as blue dots and predicted data as circles.

the observed change from undeveloped to developed cells to estimate the coefficients of the multilevel logistic regression. The core of this module is a script in the $\mathrm{R}$ language ( $\mathrm{R}$ Development Core Team, 2008), which uses the package lme4 (Bates et al., 2015) for fitting generalized linear mixed-effects models and the package MuMIn (Barto, 2015) for automatic model selection. The output file is a plain text file with tab-separated regression coefficients. This script is wrapped in Python for more seamless processing and chaining of modules. The coupling between $\mathrm{R}$, Python and GRASS GIS is intentionally very loose to make the workflow possible in the Windows environment where some of the other, more elegant, options such as $\mathrm{rpy} 2^{3}$ are complicated to use. We performed stratified sampling of observed new development and predictors using GRASS GIS add-on r.sample.category. Although we developed this add-on for urban growth modeling with FUTURES, its application is much broader. In order to encourage its use in other applications we made it a general module rather than making it part of the r.futures tool set.

3.1.4 r.futures.pga is the main engine of FUTURES - it simulates urban growth using inputs from the DEMAND and POTENTIAL submodels. The patch growing algorithm (PGA) stochastically allocates a seed for new development across the development suitability surface, challenges the seed by comparing it with a random number between 0 and 1 , and then grows a discrete patch from the seed if it survives (Meentemeyer et al., 2013). This process repeats until the number of converted cells specified by DEMAND is met. The development pressure predictor and then the development suitability values are updated based on the newly developed cells. (The development suitability is computed internally from predictors and regression coefficients supplied by POTENTIAL.) We kept the original patch growing algorithm, but significantly improved its implementation to make it faster, more memory efficient and simpler to use. We replaced a custom, undocumented configuration file with a standard module interface usable from GUI or the command line, and restructured the input and output parameters and their names so that they are easy for users to understand. We used efficient GRASS GIS libraries for reading and writing raster data, which minimized the

\footnotetext{
${ }^{3}$ rpy2 is a Python package for using R from Python (http://rpy2. bitbucket.org/)
} 
time needed to initialize the simulation. FUTURES now reads rasters in GRASS's native format instead of ASCII files. This decreased the time needed for model initialization from several minutes to several seconds for a region with tens of millions of cells. Furthermore, we replaced the static allocation of internal structures with dynamic allocation and reduced the overall memory requirements so that FUTURES could run on large regions with tens or hundreds of counties as well as smaller areas like our case study. Finally, through the use of appropriate programming techniques, such as binary search, we significantly increased the speed of the algorithm.

3.1.5 r.futures.calib We developed a dedicated Python module for calibrating patch sizes and shapes that runs the module r.futures.pga with different combinations of patch parameters and outputs a table with scores for each combination of patch parameters. The simulation is run multiple times for each combination to account for the stochasticity of the model. To speed up the calibration process r.futures.calib can take advantage of multiple computer cores.

\section{CASE STUDY}

To demonstrate how the new FUTURES framework can be used to simulate urban growth, we present a case study for Asheville metropolitan area located in the Blue Ridge Mountains in the west of North Carolina, USA. The region consists of five counties with total area of $6,271 \mathrm{~km}^{2}$ and around 477,000 people based on 2014 population estimates. It is characterized by rapid population growth around Asheville, the largest city of the region. New development is constrained by the steep mountainous terrain and large national and state parks. We simulate urban growth from 2012 to 2030 using publicly available data, including the USGS's National Land Cover Database (NLCD) (Homer et al., 2015, Fry et al., 2011, Homer et al., 2007, Fry et al., 2009), past estimates and future projections of county populations (NCOSBM, 2015), boundaries and roads provided by the United States Census Bureau's database (TIGER) and a digital elevation model from the National Elevation Dataset (NED) distributed by the USGS.

\subsection{Approach}

There are several steps required to run the FUTURES simulation:

- Preprocess the data.

- Estimate per capita land consumption controlling the total area of converted land.

- Derive the development suitability statistical model to control where the new development happens.

- Calibrate patch size and shape.

- Run the urban growth simulation.

4.1.1 Data preparation The core input data for urban growth modeling with FUTURES is a timeseries of land cover maps, which can be derived by various methods from satellite imagery. In this study we used the 2001, 2006 and 2011 NLCD Land Cover products and 1992/2001 Retrofit Land Cover Change product to derive a 30-meter binary representation of developed areas. We excluded national and state parks, water bodies and wetlands from further analysis. We used NLCD products that are available for the contiguous USA so that this study and its workflow would be easier to reproduce and apply to other study areas. We obtained population statistics from the North Carolina Office of State Budget and Management, which are based on 2000 and 2010 censuses and include past as well as future estimates of population per county for each year up to 2035. Data for the 5 counties studied were extracted and formatted as a comma-separated values (CSV) file.

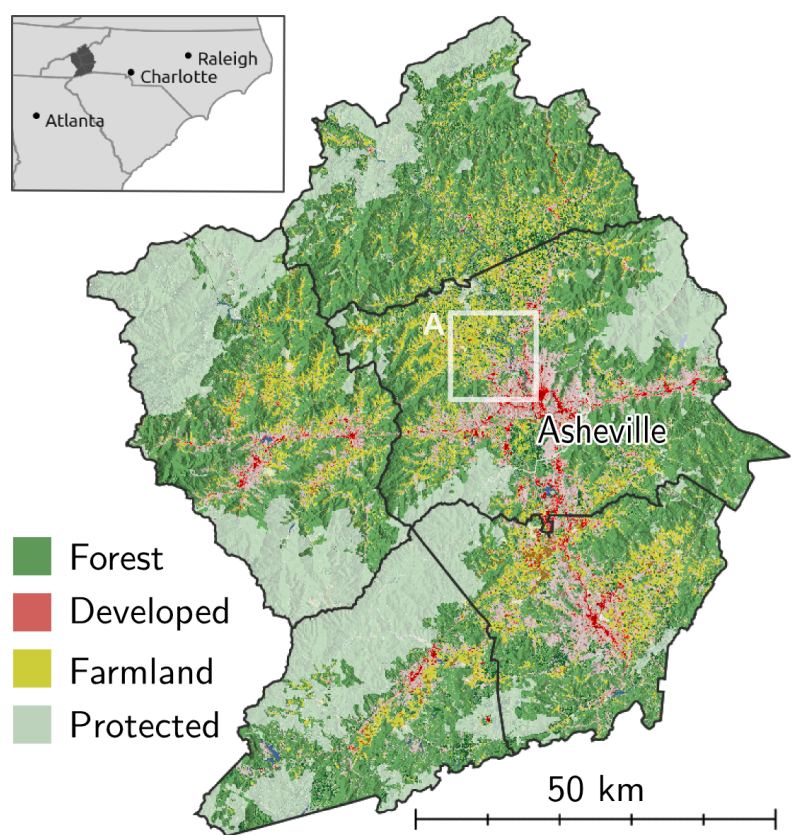

Figure 4: 2011 land cover (Homer et al., 2015) and protected areas (Anderson and Sheldon, 2011) in the Asheville metropolitan area in the west of North Carolina, USA. Inset A is used in Figure 5.

4.1.2 DEMAND We derived the relation between population and land consumption from the series of binary rasters of developed areas and population statistics to model how much land will be developed each year of the simulation. Using the module r.futures.demand we explored different curve fitting methods and derived the per capita land consumption from period 1992 through 2011, which was characterized by population growth with decreasing demand for land per person over time. We expect similarly low rates of per capita land consumption in the following years because development is restricted by the mountainous terrain and large protected areas. Based on RMSE and visual inspection of the plots created by r.futures.demand we selected either linear or logarithmic relations for each county, where the function coefficients were automatically determined using linear regression and non-linear least squares optimization in r.futures.demand (Figure 3).

4.1.3 POTENTIAL We used multilevel logistic regression to predict where new development happens based on environmental, infrastructural and socio-economic site suitability predictors. Using r.sample.category we sampled predictors on 8000 randomly selected locations and estimated the model coefficients using the $\mathrm{R}$ package lme4 integrated into the module r.futures.potential. The sample points were stratified by the response variable where new sites developed since 1992 have a value of 1 and sites that are undeveloped in 2011 have a value of 0 . We included counties as the group level indicator in the multilevel model to account for differences across jurisdictional boundaries. From the initial list of hypothesized predictors (slope, distance to water, protected areas, interchanges, travel time to cities and towns, forest occurrence and road density) we identified a set of predictors (Table 1) resulting in a model with the lowest AIC (Akaike information criterion) score. We verified the robustness of the selected predictors by repeating the random sampling and model selection process multiple times. In addition to these predictors, we included also development pressure, a special, dynamic predictor that is updated during the simulation based on new simulated development to enable positive feedback. We computed the initial development pressure raster with r.futures.devpressure; its subse- 
a) sprawl

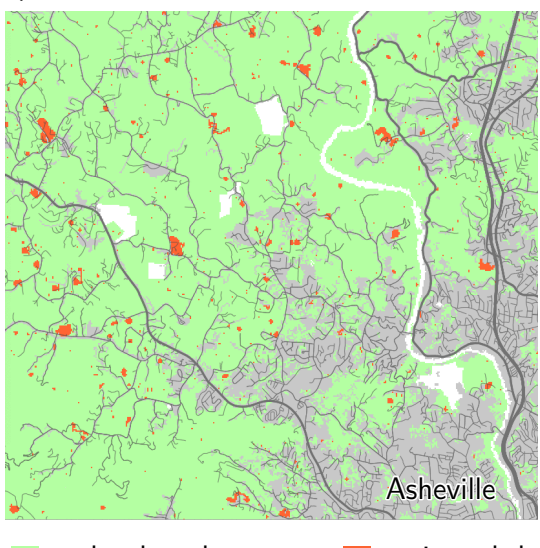

b) status quo

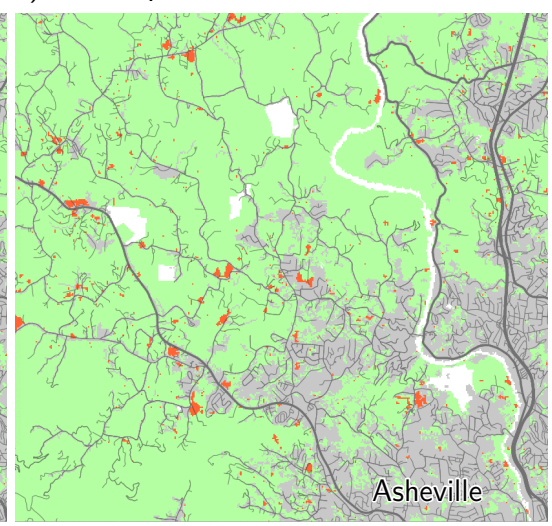

undeveloped

projected development

- roads

c) infill

Figure 5: Results of three realizations of multiple stochastic runs with different scenarios. Depending on the scenario, simulated development is more diffuse (a) or more compact (c).

quent updates are performed in memory during the simulation.

\begin{tabular}{lrr}
\hline Predictors & Estimate* & Std. Error \\
\hline Intercept (varies by county) & -2.593 & 0.269 \\
Development pressure & 0.058 & 0.005 \\
Road density & 0.118 & 0.007 \\
Percentage of forest & -0.013 & 0.002 \\
Distance to protected areas & -0.140 & 0.039 \\
Distance to water bodies & -0.148 & 0.022 \\
\hline
\end{tabular}

Table 1: List of selected predictors and estimated coefficients for site suitability model

4.1.4 Patch calibration Prior to running the urban growth simulation implemented in rfutures.pga we calibrated the input patch compactness and size to match the simulated patterns with the observed patterns from 1992 to 2011. Since calibration is a time consuming process, we ran the module r.futures.calib for Buncombe county and applied the results to the rest of our study region. We choose Buncombe County which includes the city of Asheville because it is where most new development will occur. For each combination of patch parameters we compared the patch characteristics averaged from 20 runs of the urban growth simulation with the known patches. Based on the score we selected patch parameters resulting in high compactness which is expected for mountainous regions.

4.1.5 Urban growth simulation Having collected all necessary input data, we ran rfutures.pga with a 1 year time step until 2035 for the entire study region at $30 \mathrm{~m}$ resolution. To account for different future policies regarding new development, we explored scenarios altering the site suitability to encourage infill or sprawl by changing incentive_power parameter of r.futures.pga. This value transforms the probability $p$ a cell is developed to $p^{x}$ where $x=1$ represents status quo, higher values of $x$ result in infill and lower values in sprawl. In addition to the status quo we simulated scenarios with $x$ equals $0.25,0.5,2$ and 4 . We repeated each scenario 50 times to account for the model's stochastic behavior.

\subsection{Results}

The resulting development patterns of three realizations of the random runs are visible in Figure 5 for the status quo, infill scenario $(x=4)$ and sprawl scenario $(x=0.25)$. The simulated patches realistically mimic the current patches of development in shape and size and are mostly, but not exclusively adjacent to roads as expected. Furthermore, we post-processed the results to study how different urban growth policies influence the loss of forest and agricultural land in the Asheville area (Figure 6) by averaging the loss of both land use categories over the 50 runs. In all scenarios, forest is more affected by future development than farmland. The extreme case of urban sprawl results in twice as much forest as farmland being developed. Status quo scenario leads to the smallest difference between the areas converted to forest and farmland. Interestingly, infill scenario develops the forested area in a similar way as sprawl does, which is not surprising considering developed areas are largely surrounded by forest patches.

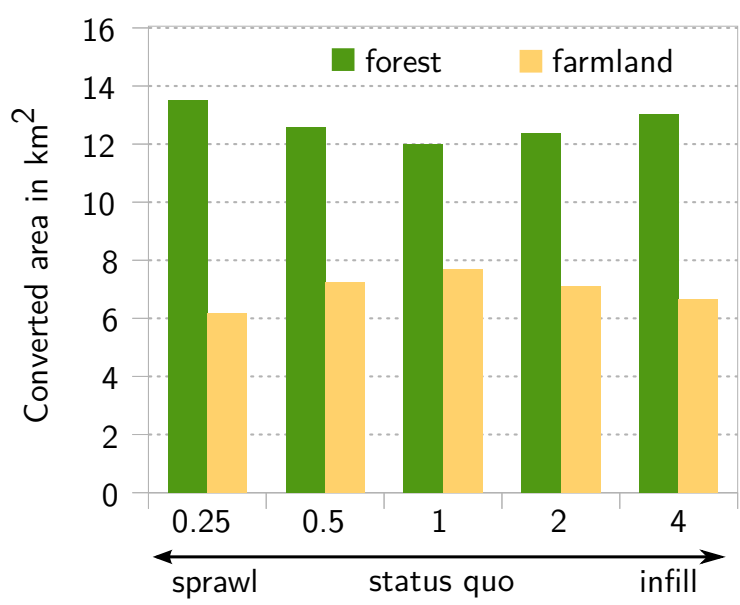

Figure 6: Area in $\mathrm{km}^{2}$ of converted land from forest (green) and farmland (yellow) to urban differs for urban sprawl and infill scenarios. Numbers 0.25 to 4 represent the exponent $x$ which transforms development probability $p$ to $p^{x}$.

Table 2 shows the computational resources necessary for running this case study and compares the time and memory requirements with the original implementation of FUTURES. Note that our study area is fairly small (12 million cells) and when applied to larger regions with more projected development, the expected speed gain is even more significant as we changed the complexity of one of the core algorithms from linear to logarithmic. Because the individual stochastic runs are independent of each other this simulation is an "embarrassingly parallel" problem (Herlihy and 
Shavit, 2012) in which the computation can easily be distributed across multiple computer cores.

\begin{tabular}{lccc}
\hline FUTURES version & memory & $1 \mathrm{run}$ & all runs (250) \\
\hline original & $1.7 \mathrm{~GB}$ & $60 \mathrm{~s}$ & $4 \mathrm{~h} 10 \mathrm{~min}$ \\
r.futures & $0.86 \mathrm{~GB}$ & $19 \mathrm{~s}$ & $1 \mathrm{~h} 20 \mathrm{~min}$ \\
\hline
\end{tabular}

Table 2: Time and memory needed to run the simulations with the old version of FUTURES and the new r.futures implemented in GRASS GIS on a laptop with 64-bit Ubuntu 14.04 LTS, Intel

Core i7-4760HQ @ 2.10GHz using 1 CPU and running on external hard drive.

The input data and instructions to run the model are available as part of material developed for the US-IALE 2016 Annual Meeting workshop on FUTURES ${ }^{4}$.

\section{DISCUSSION}

The new FUTURES framework is split into independent GRASS GIS modules so that the modeling workflow is flexible and extendable. By using standardized inputs and outputs (raster layers and CSV files) and described interface we allow FUTURES' users to replace DEMAND and POTENTIAL implementations by their own tools, which may be better suited to the characteristics and datasets available for their study systems. We ran all previous studies on county level at $30 \mathrm{~m}$ resolution. FUTURES, however, can be applied to larger or smaller scales as long as there is data available and the patch characteristics are properly calibrated. Future research will explore nested scales in order to address the different scales of the population data and the spatial drivers of land change.

\section{CONCLUSION}

We presented a new, open source version of the FUTURES urban growth model that is integrated into GRASS GIS, opening new possibilities for environmental scientists and urban planners to project and understand the impacts of urbanization at relevant ecological and decision-making scales. Integration into GRASS GIS allowed us to make FUTURES more efficient, simple to use and transparent. With documented code running on all platforms, FUTURES can now be easily tested and applied to study sites at local to megaregional scales. We illustrated how FUTURES can be used in a small case study of the Asheville metropolitan area. We also provided the instructions and data needed to reproduce this study as a step towards more reproducible research in land change science.

\section{ACKNOWLEDGEMENTS}

We would like to thank Monica Dorning and Douglas Shoemaker for discussing with us the original model implementation, and Brian Pickard and Georgina Sanchez for testing new FUTURES implementation.

\section{REFERENCES}

Alberti, M., 2005. The effects of urban patterns on ecosystem function. International regional science review 28(2), pp. 168 192.

\footnotetext{
${ }^{4}$ https://grasswiki.osgeo.org/wiki/Workshop_on_urban_ growth_modeling_with_FUTURES
}

Anderson, M. and Sheldon, A. O., 2011. Conservation Status of Fish, Wildlife, and Natural Habitats in the Northeast Landscape: Implementation of the Northeast Monitoring Framework. Technical report, The Nature Conservancy, Eastern Conservation Science.

Barto, K., 2015. MuMIn: Multi-Model Inference. R package version 1.15.1.

Bates, D., Maechler, M., Bolker, B. and Walker, S., 2015. lme4: Linear mixed-effects models using Eigen and S4. R package version 1.1-9.

Chaudhuri, G. and Clarke, K. C., 2013. The sleuth land use change model: A review. International Journal of Environmental Resources Research 1(1), pp. 88-104.

Chemin, Y., Petras, V., Petrasova, A., Landa, M., Gebbert, S., Zambelli, P., Neteler, M., Löwe, P. and Di Leo, M., 2015. GRASS GIS: a peer-reviewed scientific platform and future research repository. In: Geophysical Research Abstracts, Vol. 17, p. 8314 .

Dorning, M. A., Koch, J., Shoemaker, D. A. and Meentemeyer, R. K., 2015. Simulating urbanization scenarios reveals tradeoffs between conservation planning strategies. Landscape and Urban Planning 136, pp. 28-39.

Easterbrook, S. M., 2014. Open code for open science? Nature Geoscience 7(11), pp. 779-781.

Fry, J., Coan, M., Homer, C., Meyer, D. and Wickham, J., 2009. Completion of the National Land Cover Database (NLCD) 1992 2001 Land Cover Change Retrofit Product. Technical report, U.S. Geological Survey Open-File Report 20081379.

Fry, J., Xian, G., Jin, S., Dewitz, J., Homer, C., Yang, L., Barnes, C., Herold, N. and Wickham, J., 2011. Completion of the 2006 National Land Cover Database for the Conterminous United States. PE\&RS 77(9), pp. 858-864.

Herlihy, M. and Shavit, N., 2012. The Art of Multiprocessor Programming, Revised Reprint. Elsevier.

Homer, C., Dewitz, J., Fry, J., Coan, M., Hossain, N., Larson, C., Herold, N., McKerrow, A., VanDriel, J. and Wickham, J., 2007. Completion of the 2001 National Land Cover Database for the Conterminous United States. Photogrammetric Engineering and Remote Sensing 73(4), pp. 337-341.

Homer, C., Dewitz, J., Yang, L., Jin, S., Danielson, P., Xian, G., Coulston, J., Herold, N., Wickham, J., and Megown, K., 2015. Completion of the 2011 National Land Cover Database for the conterminous United States - Representing a decade of land cover change information. Photogrammetric Engineering and Remote Sensing 81(5), pp. 345-354.

Jantz, C. A. and Goetz, S. J., 2005. Analysis of scale dependencies in an urban land-use-change model. International Journal of Geographical Information Science 19(2), pp. 217-241.

McGarigal, K., Cushman, S. and Ene, E., 2012. FRAGSTATS v4: spatial pattern analysis program for categorical and continuous maps. Computer software program produced by the authors at the University of Massachusetts, Amherst.

Meentemeyer, R. K., Tang, W., Dorning, M. A., Vogler, J. B., Cunniffe, N. J. and Shoemaker, D. A., 2013. FUTURES: Multilevel Simulations of Emerging UrbanRural Landscape Structure Using a Stochastic Patch-Growing Algorithm. Annals of the Association of American Geographers 103(4), pp. 785-807.

NCOSBM, 2015. County/State Population Projections. 
Neteler, M. and Mitasova, H., 2008. Open Source GIS: A GRASS GIS Approach. Vol. 773, Springer, New York.

Pickard, B. R., Van Berkel, D., Petrasova, A. and Meentemeyer, R. K., in prep. Future patterns of urbanization reveal trade-offs among ecosystem services.

R Development Core Team, 2008. R: A Language and Environment for Statistical Computing. R Foundation for Statistical Computing, Vienna, Austria. ISBN 3-900051-07-0.

Shoemaker, D. A., 2016. The role of spatial heterogeneity and urban pattern in modulating ecosystem services. Doctoral dissertation, North Carolina State University. Retrieved from http://www.lib.ncsu.edu/resolver/1840.16/11033.

Sohl, T. L., Sayler, K. L., Drummond, M. A. and Loveland, T. R., 2007. The FORE-SCE model: a practical approach for projecting land cover change using scenario-based modeling. Journal of Land Use Science 2(2), pp. 103-126.

Verburg, P. H., Soepboer, W., Veldkamp, A., Limpiada, R., Espaldon, V. and Mastura, S. S., 2002. Modeling the spatial dynamics of regional land use: the CLUE-S model. Environmental management 30(3), pp. 391-405.

Waddell, P., 2002. UrbanSim: Modeling urban development for land use, transportation, and environmental planning. Journal of the American Planning Association 68(3), pp. 297-314.

Wilson, G., Aruliah, D., Brown, C. T., Hong, N. P. C., Davis, M., Guy, R. T., Haddock, S. H., Huff, K. D., Mitchell, I. M., Plumbley, M. D. et al., 2014. Best practices for scientific computing. PLoS Biol 12(1), pp. e1001745. 